\title{
Quiste Broncogénico de Localización Poco Habitual: Reporte de 2 Casos y Revisión de la Literatura
}

\author{
Bronchogenic Cyst of Unusual Location: Report of Two Cases and Review of the Literature
}

\author{
Enrique Bellolio J.; Oscar Tapia; Pablo Guzman \& Miguel Villaseca H.
}

BELLOLIO, J. E.; TAPIA, O.; GUZMAN, P. \& VILLASECA, H. M. Quiste broncogénico de localización poco habitual: reporte de 2 casos y revisión de la literatura. Int. J. Morphol., 29(4):1313-1316, 2011.

RESUMEN: El quiste broncogénico es un remanente del desarrollo de traquea y bronquios. Alteraciones en la migración de células durante el desarrollo, permite que estos grupos originen quistes revestidos por epitelio respiratorio. La ubicación más habitual es la intratoráxica, dentro de ella, la intrapulmonar es la más frecuente, seguida de la mediastínica. El diagnóstico definitivo se establece mediante la biopsia diferida. Es una patología poco frecuente y las presentaciones extra torácicas son muy infrecuentes, por lo que compartimos dos casos, uno de ubicación sublingual y otro de ubicación subcutánea en zona escapular.

PALABRAS CLAVE: Quiste broncogénico; Quiste bronquial; Quiste congénito.

\section{INTRODUCCIÓN}

El quiste broncogénico es un remanente del desarrollo de tráquea y bronquios. Desde la $5^{\text {a }}$ semana de gestación comienza el desarrollo a partir de la separación del intestino anterior, que da origen al esbozo pulmonar y bronquial. Del esbozo pulmonar se desarrolla la tráquea, la que se fusiona con los bronquios principales derivados de los esbozos bronquiales laterales (Aktogu et al., 1996). Alteraciones en la migración de células durante el desarrollo, permite que estos grupos originen quistes revestidos por epitelio respiratorio. La ubicación más habitual es la intratoráxica, dentro de ella, la intrapulmonar es la más frecuente, seguida de la mediastínica. También se han descrito en otras ubicaciones, destacando en o bajo el diafragma, pericardio, cuello, retrofarínge, dermis del hombro y región escapular (Kanemitsu et al., 1999; Pul \& Pul, 1994; Jona et al., 1995; Jacob et al., 2007; Pérez et al., 2008).

Dado que es una patología poco frecuente y las presentaciones extra torácicas son aún más infrecuentes es que presentamos dos casos que se presentaron durante el año 2009 en la unidad de anatomía patológica del Hospital Dr. Hernán Henríquez Aravena de Temuco, Chile.

\section{CASO CLÍNICO 1}

Paciente de sexo femenino de 32 años, que consultó por aumento de volumen progresivo submentoniano de 6 meses de evolución, acompañado de disfagia y dificultad respiratoria en decúbito. Se realizó ecografía que informó quiste unilocular de paredes finas de $5 \times 7 \times 4 \mathrm{~cm}$ submentoniano central profundo a los planos musculares interpretado como quiste con aspecto de ránula sublingual (Fig. 1). Se realizó extirpación quirúrgica completa de la lesión. El examen anatomopatológico de la pieza quirúrgica destacó formación quística abierta y vaciada de aproximadamente $4 \mathrm{~cm}$ de eje mayor. Cavidad quística unilocular en partes levemente irregular con contenido serohemático. Al examen microscópico se observa lesión quística con revestimiento epitelial de tipo columnar ciliado seudoestratificado de tipo respiratorio (Fig. 2).

\section{CASO CLÍNICO 2}

Paciente de sexo masculino de 14 años que consultó por aumento de volumen en zona escapular. Al examen clíni- 


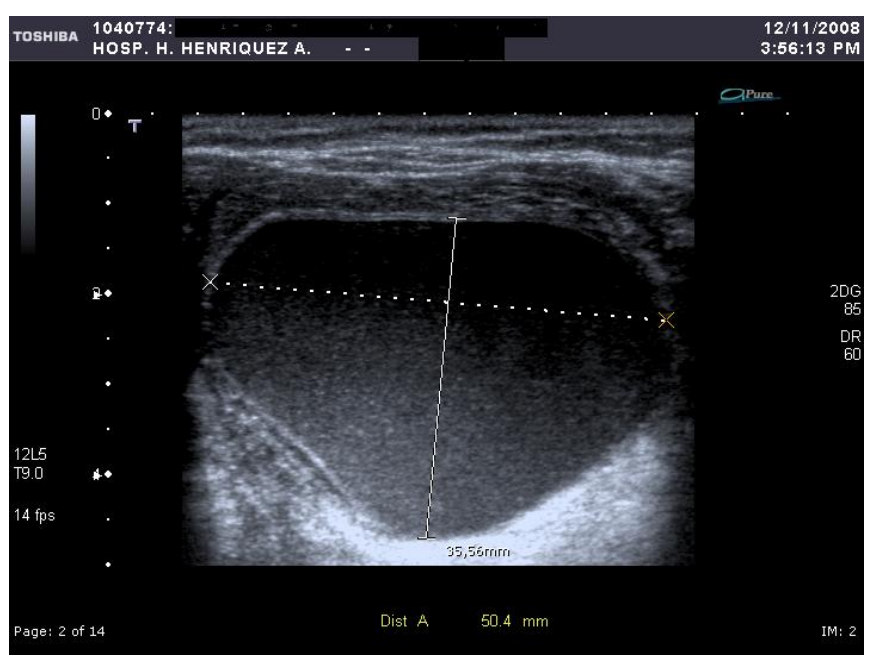

Fig. 1. Ecografía se observa quiste unilocular submentoniano central con aspecto de ránula sublingual.

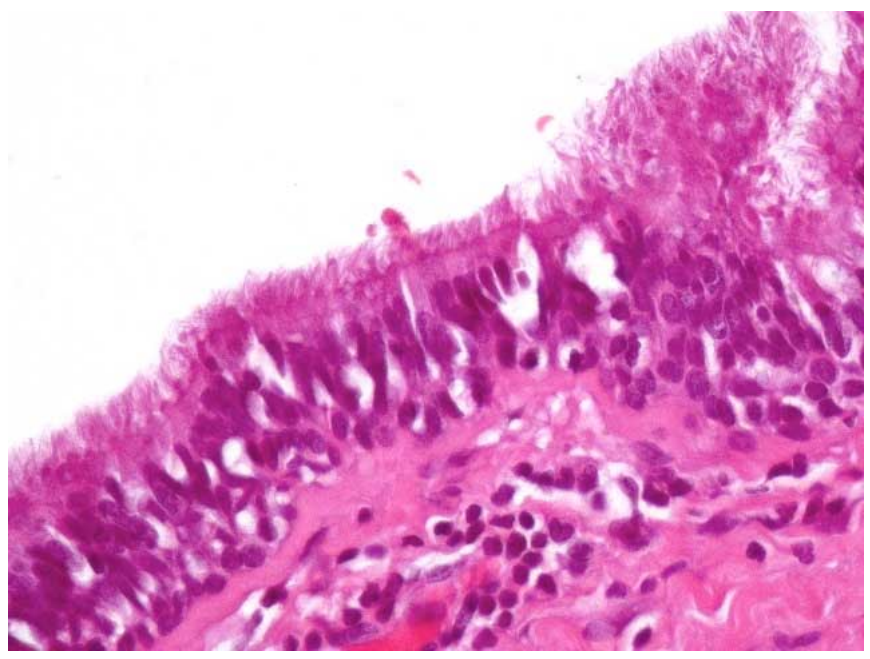

Fig. 2. Se observa lesión quística con revestimiento epitelial columnar ciliado seudoestratificado de tipo respiratorio.HE

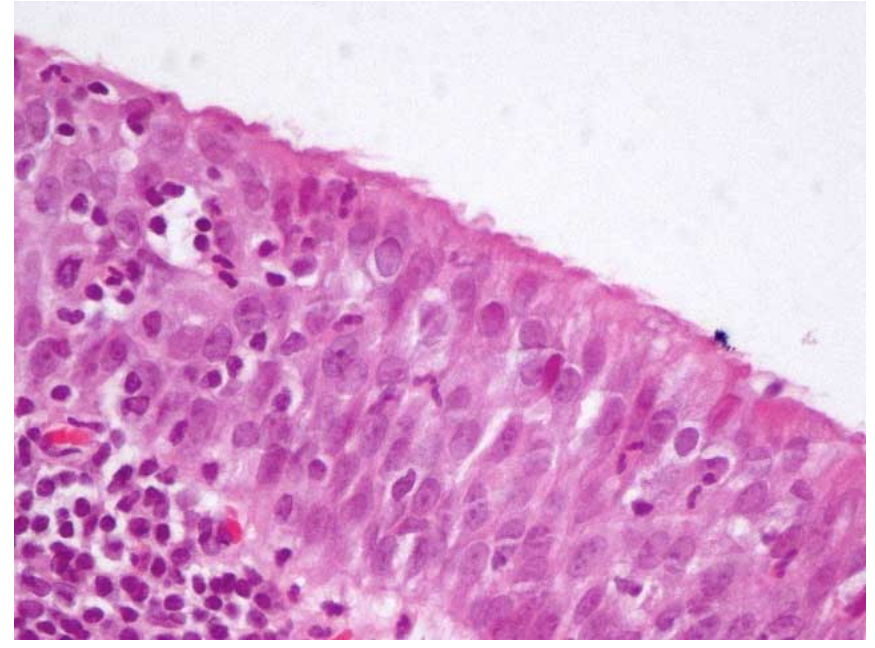

Fig. 3. Se observa lesión quística con revestimiento epitelial columnar ciliado pseudoestratificado de tipo respiratorio.HE co se describe lesión quística fluctuante de $2 \mathrm{~cm}$ de diámetro mayor, no adherido a planos profundos. Se realizó extirpación completa de la lesión.

El examen anatomopatológico de la pieza quirúrgica destaca fragmento de piel que a nivel de dermis presenta lesión quística unilocular de 1,2 cm de diámetro. Al examen microscópico se observa lesión quística con revestimiento epitelial de tipo columnar ciliado pseudoestratificado de tipo respiratorio (Fig. 3).

\section{DISCUSIÓN}

La presencia de quistes broncogénicos fuera de la cavidad torácica es infrecuente, pero el reporte de localizaciones extratorácicas se ha incrementado en la literatura en los últimos 10 años.

Se presentan predominantemente en la infancia, pero pueden diagnosticarse a cualquier edad, no existiendo un predominio por género. La presentación clínica generalmente es asintomática, presentándose en ocasiones, como un hallazgo en la radiografía de tórax (Cioffi et al., 1998).

La presentación subcutánea es de una masa quística, no adherida, de tamaño variable. Pueden complicarse con infección, llegando a la formación de abscesos que pueden fistulizar (Newkirk et al., 2004).

Los exámenes complementarios de utilidad para precisar el diagnóstico son la tomografía axial computada de corte fino y ecotomografía de partes blandas, variando la hipótesis diagnóstica según la ubicación anatómica.

Histológicamente corresponden a quistes uniloculares, revestidos por epitelio columnar o cúbico ciliado con o sin pseudoestratificación. Pueden presentar metaplasia escamosa focal o extensa y presencia de infiltrado inflamatorio crónico leve. En la pared del quiste es frecuente encontrar fibras musculares lisas, estructuras glandulares mucosas y la presencia de cartílago, este último puede no estar presente (Leslie \& Wick, 2005; Colby et al., 1995; Elder, 2009).

En los dos casos presentados, el diagnóstico clínico no incluyo en el diferencial el de quiste broncogénico dada su localización anatómica y forma de presentación. La presentación en la región oral de los quistes broncogénicos se ha reportado en 6 artículos y en todos los casos fue en edad pediátrica (Kim et al., 2001; Benhammou et al., 2006; EchCherif El Kettani \& Dafiri, 2004; Obiechina et al., 1999; 
Bailey, 1982; Boué et al., 1994). La evolución, localización submentoniana y examen ecográfico del caso 1 hizo sospechar una ránula sublingual, que es la patología más frecuente en esta localización y grupo etáreo. Pero la histología fue la que entrego el diagnóstico definitivo. Esta paciente tuvo una buena evolución tras la cirugía y no ha presentado recidiva en 24 meses de seguimiento.

La presentación cutánea en zona peri-escapular tiene una incidencia estimada de 1 en 42.000 a 1 en 68.000 (Schouten van der Velden et al., 2006). Se han reportado 13 casos en los últimos 5 años. (Ozel et al., 2005; Kundal et al., 2008).

En nuestro caso la lesión fue pesquisada en el período de recién nacido y dado su aumento de tamaño y complicación con infección se extirpó finalmente a los 14 años.
La evaluación y procedimiento diagnóstico de este tipo de lesiones debe siempre incluir un estudio imagenológico con ecotomografía para evaluar las características de la lesión, algunos han reportado la mayor utilidad de la resonancia nuclear magnética en estas lesiones (Suen et al., 1993), pero fue solo un reporte. Lamentablemente no hay hallazgos imagenológicos específicos que permitan sospechar el quiste broncogénico en localizaciones extra torácicas.

Para concluir podemos señalar que el diagnóstico de esta entidad es histopatológico, su ubicación anatómica es muy variada y debe ser sospechado principalmente en población pediátrica.

BELLOLIO, J. E.; TAPIA, O.; GUZMAN, P. \& VILLASECA, H. M. Bronchogenic cyst of unusual location: report of two cases and review of the literature. Int. J. Morphol., 29(4):1313-1316, 2011.

SUMMARY: The bronchogenic cyst is a remanent of the development of the tracheobronchial tree. Alterations in cell migration during development cause the formation of cyst lining by respiratory epithelium. They are usually located in the thorax in lung and mediastinum. The definite diagnosis is realized with the pathology report. This is an uncommon pathological condition and the extra- thoracic forms are very unusual. We present two cases, one of which is of sublingual location and the other of subcutaneous location at the scapular site.

KEY WORDS: Bronchogenic cyst; Bronchial cyst; Congenital cyst.

\section{REFERENCIAS BIBLIOGRAFICAS}

Aktogu, S.; Yuncu, G.; Halilçolar, H.; Ermete, S. \& Buduneli, T. Bronchogenic cysts: clinicopathological presentation and treatment. Eur. Respir. J., 9(10):2017-21, 1996.

Bailey, B. M. A detached bronchogenic cyst occurring in the tongue of a neonate. Br. J. Oral Surg., 20(4):288-93, 1982.

Benhammou, A.; Benyoussef, N.; Bencheikhe, R.; Benbouzid, M. A.; Boulaich, M.; Essakali, L. \& Kzadri, M. Bronchogenic cyst of the tongue. Ann. Otolaryngol. Chir. Cervicofac., 123(3):148-51, 2006.

Boué, D. R.; Smith, G. A. \& Krous, H. F. Lingual bronchogenic cyst in a child: an unusual site of presentation. Pediatr. Pathol., 14(2):201-5, 1994.

Colby, T.; Koss, M. \& Travis, W. Tumor of the Lower Respiratory Tract. In: Atlas of Tumor Pathology Armed Forces Institute Of Pathology, Third Series, Fascicle 13, 1995.

Cioffi, U.; Bonavina, L.; De Simone, M.; Santambrogio, L.; Pavoni, G.; Testori, A. \& Peracchia, A. Presentation and surgical management of bronchogenic and esophageal duplication cysts in adults. Chest, 113(6):1492-6, 1998.
Elder, D. E. Lever's Histopathology of the skin. Lippincott Williams \& Wilkins, 2009.

Ech-Cherif El Kettani, N. \& Dafiri, R. Bronchogenic cyst of the tongue: a very rare localization. J. Radiol., 85:1736-8, 2004.

Jacob, J. K.; George, S.; Roy, B. R.; Preethi, S.; Ranjith, V. T. \& Pappachan, J. M. Retropharyngeal bronchogenic cyst. Otolaryngol. Head Neck Surg., 136:1025-6, 2007.

Jona, J. Z. Extramediastinal bronchogenic cyst in children. Pediatr. Dermatol., 12:304-6, 1995.

Kanemitsu, Y.; Nakayama, H.; Asamura, H.; Kondo, H.; Tsuchiya, R. \& Naruke, T. Clinical features and management of bronchogenic cyst: report of 17 cases. Surg. Today, 29(11):1201-5, 1999.

Kim, S. H.; Han, M. H.; Park, S. W. \& Chang, K. H. Radiologicpathologic correlation of unusual lingual masses: Part I: congenital lesions. Korean J. Radiol., 2(1):37-41, 2001.

Kundal, A. K.; Zargar, N. U. \& Krishna, A. Scapular bronchogenic cyst. J. Indian Assoc. Pediatr. Surg., 13(4):147-8, 2008. 
BELLOLIO, J. E.; TAPIA, O.; GUZMAN, P. \& VILLASECA, H. M. Quiste broncogénico de localización poco habitual: reporte de 2 casos y revisión de la literatura. Int. J. Morphol., 29(4):1313-1316, 2011.

Newkirk, K.; Tassler, A.; Krowiak, E. \& Deeb, Z. Bronchogenic cysts of the neck in adults. Ann. Otol. Rhinol. Laryngol., 113:691-5, 2004

Leslie, K. \& Wick, M. Practical Pulmonary Pathology. Elsiever, 2005, pp. 51-70.

Obiechina, A. E.; Arotiba, J. T. \& Ogunbiyi, J. O. Coexisting congenital sublingual dermoid and bronchogenic cyst. Br. J. Oral Maxillofac. Surg., 37(1):58-60, 1999.

Ozel, S. K.; Kazez, A.; Koseogullari, A. A. \& Akpolat, N. Scapular bronchogenic cysts in children: case report and review of the literature. Pediatr. Surg. Int., 21(10):843-5, 2005.

Pérez, J.; Gabrielli, M.; Felmer, O. \& Carrasco, C. Quiste broncogénico cervical. Reporte de un caso. Rev. Chil. Cir., (60):51-4, 2008.

Pul, N. \& Pul, M. Bronchogenic cyst of the scapular area in an infant: case report and review of the literature. J. Am. Acad. Dermatol., 31(1):120-2, 1994.

Schouten van der Velden, A. P.; Severijnen, R. S. \& Wobbes, T. A bronchogenic cyst under the scapula with a fistula on the back. Pediatr. Surg. Int., 22:857-60, 2006.

Suen, H. C.; Mathisen, D. J.; Grillo, H. C.; LeBlanc, J.; McLoud, T. C.; Moncure, A. C. \& Hilgenberg, A. D. Surgical management and radiological characteristics of bronchogenic cyst. Ann. Thorac. Surg., 55:476-81, 1993.

\author{
Dirección para correspondencia: \\ Enrique Bellolio J. \\ Depto. Anatomía Patológica \\ Facultad de Medicina \\ Universidad de La Frontera \\ Casilla 54-D \\ Temuco \\ CHILE \\ Teléfono: 045-325735 \\ Fax: 045-325735 \\ E-mail: ebellolio@gmail.com \\ Recibido : 26-07-2011 \\ Aceptado: 28-08-2011
}

